\title{
$\angle S$ Research Square \\ The Impact of COVID-19 On Emergency Visits For Back And Neck Pain
}

\author{
Nissim Ohana \\ Meir Medical Center \\ Itzhak Engel \\ Meir Medical Center \\ Yuval Baruch \\ Meir Medical Center
}

Daniel Benharroch ( $\nabla$ danielbenharroch1@gmail.com )

Soroka University Medical Center https://orcid.org/0000-0002-5178-5851

Dimitri Sheinis

Soroka University Medical Center

\section{Research Article}

Keywords: COVID-19, lockdown, low back pain, neck pain, emergency department stays

Posted Date: January 3rd, 2022

DOI: https://doi.org/10.21203/rs.3.rs-1156526/v1

License: (c) (1) This work is licensed under a Creative Commons Attribution 4.0 International License.

Read Full License 


\section{Abstract}

Purpose: To assess the rate of visits to the emergency department of our medical center concerning low back or neck pain, as a factor of COVID-19 confinements.

Methods: The study period was a 30-weeks interval during the COVID-19 pandemic contrasted by a similar stretch in the year preceding the epidemic. The visits to the Emergency Department, prompted by low back or neck pain, were recorded prior and during lockdowns of the pandemic. The significance of the confinements for the development of the pain syndromes was evaluated.

Results: A total of 1530 patients with newly diagnosed back or neck pain were enrolled. Most patients visited our emergency department for low back pain, commonly those older than 60 years. No significant gender variance was disclosed, though most visits of females were for low back pain. Low back pain presentations were curbed following confinement, but the rate of stays for neck pain had swelled by more than $10 \%$. Despite back pain predominance, visits for neck pain persisted. Before COVID-19, the average weekly number of Emergency Department visits was 38.5. This was followed by sharp drops during COVID-19 lockdown (mean different=-22.2, 95\%Cl=-28.7, -15.7, $p<0.001$ ) (not significant).

Conclusions: COVID-19 lockdowns have a significant impact on the Emergency Department's presentations due to back and neck pain. A higher rate of presentation for back pain compared to neck pain is probably related to COVID-19, without being affected directly by the SARS-CoV-2: confinementinduced immobility might instigate musculoskeletal sequels, which may be attributed to stress or other psycho-social afflictions.

\section{Introduction}

Low back pain (LBP) and neck pain (NP) are common complaints which tend to recur with aging. The etiology varies from non-specific causes to specific disease entities, such as herniated disc, infectious diseases or tumors [1, 2]. The mode of expression is therefore diversified. In addition to comparing LBP and NP, we intend to assess these complaints as they develop in different categories of patients [3-5].

Remarkably, we were able to scrutinize the behavior of two conditions which, a priori should exhibit two cohorts with LBP and NP patients, display a similar clinical picture, and a parallel clinical outcome [6]. Both subsets were composed of adult patients with above-mentioned syndromes. Both cohorts were free from associated COVID-19, since a pre-requisite for ED attendance was the lack of any respiratory or febrile complaint. Only patients who were negative for the virus were treated at the ED. Patients with a positive SARS-CoV-2RT-PCR test were steered to the COVID-19-ED. The association between the COVID-19 confinement and the musculoskeletal conditions was highlighted $[3,5]$.

\section{Materials And Methods}


It is hypothesized that, as a consequence of the confinements implemented by the authorities to fight the spread of COVID-19, a general immobilization will develop. A secondary effect may evolve in the form of a marked limitation of presentations to the ED, among others of LBP and NP.

\section{Study design and population}

An acceptable terminology should first be established. Lockdown is employed interchangeably for confinement; ED visit (ED stay, ED sojourn) for ED presentation; low back pain for low back pain/sciatica; neck pain for neck pain/cervicalgia. The simplest terms are to be adopted whenever possible.

Back and neck pain were defined by the International Statistical Classification of Diseases and Related Health Problems, Tenth Revision (ICD-10) code (Fig. 1). All patients included in our study were SARS-CoV2-RT-PCR-negative and were taken care of in the general ED. Each patient's demographics, length of stay in the ED and diagnoses were retrieved from the hospital electronic database. No individual identifiers (e.g., name and address) were disclosed. Patients were excluded from the analysis if they were readmitted or if they were younger than 18 years. The study has been approved by the Institutional Review Board prior to data collection.

\section{Exposure and Outcome}

The study period was categorized into two groups, namely, 30 weeks before the COVID-19 pandemic lockdowns began and 30 weeks since then. The primary outcome was the mean weekly number of patients with newly identified back or neck pain. A relationship between the spinal syndromes and a reduced physical activity was displayed.

\section{Statistical analysis}

The number of patients $\mathrm{n}(\%)$ and Mean (standard deviation, SD) were used to describe continuous and categorical variables, respectively. Analysis of variance (ANOVA) or chi-squared test were used to examine differences of demographics between two groups (before and after lockdown). We applied an interrupted time-series analysis using segmented linear regression to explore the impact of COVID-19 lockdowns on the trends of ED presentations occasioned by LBP and NP [7]. In the interrupted time series analysis, $\beta_{0}$ estimated the start level of the weekly ED presentations at 1 year before lockdown (intercept); $\beta_{1}$ estimated the before-lockdown slope, which quantified the trend for the weekly ED presentations before lockdown; $\beta_{2}$ estimated the change in level at the 1 st lockdown point, which can arguably be attributed to lockdown; and $\beta_{3}$ estimated the change in slope from before to after lockdown, which quantified the difference between the prior and ensuing lockdown slopes. All analyses were performed using SAS version 9.4 (SAS Institute Inc., Cary, NC, USA). Two-sided p <0.05 was considered statistically significant.

\section{Results}


A total number of 1898 Emergency Department visits for neck and back pain were recorded in the hospital database between March 2019 to November 2020. Of these, 368 patients were excluded since they did not belong in the study period, or due to different diagnoses or missing data. Figure 2 describes the flow diagram defining the eligible patients.

Table 1. Represents the patients' weekly presentation to the ED in the period preceding the pandemic, as well as during the lockdowns. As expected, the variance between the mean number of sojourns in both periods is significantly different, with a median number of 36.5 visits a week during the year preceding the pandemic, as compared to 16 visits along its stretch.

Table 1

Weekly number of ED presentations before and during lockdowns.

\begin{tabular}{|llll|}
\hline & Before lockdown & During lockdown & P value \\
\hline Weekly ED presentations & & & $<0.001$ \\
\hline Mean (SD) & $37.1(7.1)$ & $16.5(5.7)$ & \\
\hline Median (IQR) & $36.5(31.0,42.0)$ & $16.0(25.0,50.0)$ & \\
\hline
\end{tabular}

As shown in Table $2, \beta_{0} \beta_{1} \beta_{2} \beta_{3}$ there was a significant difference between the weekly visits at the beginning of lockdowns (mean different=-22.2, 95\%Cl=-28.7,-15.7, $\mathrm{p}<0.001$ ). However, there was no significant difference in the weekly visit trend between the period preceding the pandemic and that encompassing the lockdowns (slope different $=0.19,95 \% \mathrm{Cl}=-0.08,0.45, \mathrm{p}=0.17$ ).

Table 2

Interrupted time-series analysis for the weekly number of presentations

\begin{tabular}{|llll|}
\hline & \multicolumn{2}{l|}{ ED presentations (per week) } & \\
\cline { 2 - 3 } & Coefficient & $95 \%$ Confidence interval & P value \\
\hline Presentations & & & \\
\hline Intercept $\left(\beta_{0}\right)$ & 38.5 & $33.8,43.2$ & $<0.001$ \\
\hline Trend before lockdown $\left(\beta_{1}\right)$ & -0.09 & $-0.36,0.18$ & 0.51 \\
\hline Level change after lockdown $\left(\beta_{2}\right)$ & -22.2 & $-28.7,-15.7$ & $<0.001$ \\
\hline Trend after lockdown $\left(\beta_{3}\right)$ & 0.28 & $-0.10,0.65$ & 0.16 \\
\hline Trend change after lockdown (beta 1 +beta 3) & 0.19 & $-0.08,0.45$ & 0.17 \\
\hline
\end{tabular}

Table 3 highlights the demographics and the diagnoses attributed in the ED, as they relate with the status of the lockdowns. More males were identified. The mean age was higher before the pandemic. The mean 
length of stay in ED was longer before the lockdowns. Although a sustained predominance of LBP is evident at all times, neck pain, exhibits a discordance: while the number of visits is reduced, from "before lockdowns", the rate of visits with neck pain raised from $24.4-34.1 \%$.

Table 3. The weekly number of ED visits before and during confinements.

\begin{tabular}{lccc} 
Variable & $\begin{array}{c}\text { Before lockdowns } \\
\text { (2019MAR25-2019OCT21) }\end{array}$ & $\begin{array}{c}\text { During Lockdowns } \\
(2020 \mathrm{MAR} 25-2020 \mathrm{OCT} 21)\end{array}$ & \\
\hline Gender & & & Chi-square test \\
N (Nmiss) & $1113(1)$ & $494(1)$ & 0.01 \\
M & $563(50.6 \%)$ & $284(57.5 \%)$ & \\
F & $550(49.4 \%)$ & $210(42.5 \%)$ & T test \\
Age & & & 0.01 \\
N (Nmiss) & $1113(1)$ & $494(1)$ & \\
Mean (SD) & $50.39(18.53)$ & $47.84(18.32)$ & \\
Median & 49.00 & 48.00 & \\
Q1;Q3 & $38.00 ; 65.00$ & $35.00 ; 61.00$ & \\
Min;Max & $2.00 ; 98.00$ & $0.00 ; 92.00$ & 0.003 \\
Length of stay (hour) & & & \\
N (Nmiss) & $1114(0)$ & $495(0)$ & \\
Mean (SD) & $3.80(2.01)$ & $3.50(1.82)$ & \\
Median & 3.48 & 3.23 & \\
Q1;Q3 & $2.38 ; 4.82$ & $2.27 ; 4.43$ & \\
Min;Max & $0.38 ; 16.50$ & $0.43 ; 15.43$ &
\end{tabular}

Diagnosis in emergency

Chi-square test

$\mathrm{N}$ (Nmiss)

$1114(0)$

$495(0)$

$<0.0001$

LOW BACK PAIN/SCIATI 801( $71.9 \%)$

$290(58.6 \%)$

NECK PAIN/CERVICALGI $272(24.4 \%)$

$169(34.1 \%)$

OTHER

$41(3.7 \%)$

$36(7.3 \%)$

In the first quarter of the pandemic, the number of presentations dropped significantly to 25 a week, when contrasted with 31 in the previous year. However, in the third quartile, which occurred after this Country 
had left behind it the first episode of confinement, the mean number of visits to the ED expanded to a weekly mean of 50 , like that of the parallel quartile, in the year preceding the epidemic.

Figure 3 also shows the change in the mean weekly number of presentations between the two periods (before and during COVID-19 lockdowns). Of note, with the start of lockdowns, a sharp drop in the weekly number of visits to the ED occurred. Later, after the release of the confinement, and the return to a regular mode of life, a moderate raise in the weekly number of visits to the ED was noted.

Table 4 contrasts patients with LBP $(n=1090)$ with those suffering from neck pain $(n=440)$. No significant gender variance was disclosed. LBP in males, 582 (72.4\%), was higher in comparison with females, 508 $(70.0 \%)$. Concerning neck pain in males, $222(27.6 \%)$, in contrast with females, $218(30.0 \%), p=0.30)$. Low back pain was prominent in patients older than 60 years, while NP was distributed around patients 40 to 60 years old. The stays in the ED lasted more than two hours for most LBP patients. Patients affected by LBP represented the majority during the pre-lockdown period while the rate of NP cases was higher during the pandemic. 
Table 4

Baseline characteristics of eligible patients by diagnosis (row \%)

\begin{tabular}{|llll|}
\hline Variable & $\begin{array}{l}\text { Low back pain/Sciatica } \\
(\mathbf{n}=1090)\end{array}$ & $\begin{array}{l}\text { Neck pain/Cervicalgia } \\
(\mathbf{n}=440)\end{array}$ & P value \\
\hline Gender, $\mathrm{n}(\%)$ & & $222(27.6)$ & 0.30 \\
\hline Male & $582(72.4)$ & $218(30.0)$ & \\
\hline Female & $508(70.0)$ & $46.64(18.45)$ & $<0.001$ \\
\hline Age (y), mean (SD) & $52.10(16.97)$ & & $<0.001$ \\
\hline Age group (y), n (\%) & & $17(85.0)$ & \\
\hline$<20$ & $3(15.0)$ & $170(26.6)$ & 0.02 \\
\hline $20-<40$ & $260(63.0)$ & $100(21.8)$ & 0.004 \\
\hline $40-<60$ & $468(73.4)$ & $3.58(1.99)$ & $<0.001$ \\
\hline$\geq 60$ & $359(78.2)$ & & \\
\hline Time in ER (h), Mean (SD) & $3.79(1.93)$ & $94(36.3)$ & \\
\hline Time in ER (h), $\mathrm{n}(\%)$ & & $346(27.2)$ & \\
\hline$<2 \mathrm{~h}$ & $165(63.7)$ & $271(25.3)$ & \\
\hline$\geq 2 \mathrm{~h}$ & $925(72.8)$ & & \\
\hline Lockdown, $\mathrm{n}(\%)$ & $801(74.7)$ & & \\
\hline Before & $289(63.1)$ & & \\
\hline During & & & \\
\hline Notes, Chi-square test or T test were used for testing group difference. & & \\
\hline
\end{tabular}

LBP displayed a combined decrease of numbers and rate, from 801 (74.7\%) in the prior year to 289 (63.1\%) during the COVID-19 lockdowns. In contrast, although the number of ED visits due to NP decreased from 271 to 169, the rate increased from 25.3-36.9\% (more than 10\% excess), indicating a contrast in the patients' attitude regarding their decision to visit the ED.

Table 5 exhibits a multivariable regression outlining factors associated with the diagnosis of 1090 cases of LBP in the ED. Gender was not statistically significant. The significant age group appears to be $20-<40$. The time in ED is 2 hours or greater. More cases and a higher rate of LBP were exhibited before the pandemic. 
Table 5

Multivariable logistic regression relating the visit of 1090 patients diagnosed with LBP in the ED.

\begin{tabular}{|c|c|c|}
\hline Variable & $\begin{array}{l}\text { Low back pain/Sciatica } \\
(n=1090)\end{array}$ & Odd Ratio (95\% Cl), p \\
\hline \multicolumn{3}{|c|}{ Gender, n (\%) } \\
\hline Male & $582(72.4)$ & 1.00 (ref) \\
\hline Female & $508(70.0)$ & $0.81(0.64,1.02), 0.07$ \\
\hline \multicolumn{3}{|c|}{ Age group (y), n (\%) } \\
\hline$<20$ & $3(15.0)$ & $0.06(0.02,0.20),<0.001$ \\
\hline $20-<0$ & $260(63.0)$ & $0.47(0.34,0.63),<0.001$ \\
\hline $40<60$ & $468(73.4)$ & $0.75(0.57,1.00), 0.05$ \\
\hline$\geq 60$ & $359(78.2)$ & 1.00 (ref) \\
\hline \multicolumn{3}{|c|}{ Time in ER (h), $n(\%)$} \\
\hline$<2 \mathrm{~h}$ & $165(63.7)$ & 1.00 (ref) \\
\hline$\geq 2 \mathrm{~h}$ & $925(72.8)$ & $1.37(1.02,1.83), 0.04$ \\
\hline \multicolumn{3}{|c|}{ Lockdown, n (\%) } \\
\hline Before & $801(74.7)$ & 1.00 (ref) \\
\hline During & $289(63.1)$ & $0.59(0.46,0.75),<0.001$ \\
\hline
\end{tabular}

Immobility and lack of physical activity is proposed as a potential mechanism by which back and neck pain syndromes might be established following a COVID-19 lockdowns [3, 5].

\section{Discussion}

The present study was intended to scrutinize whether the COVID-19 pandemic did change the pattern of attendance of patients with low back or neck pain to the ED. Non-specific pain in the neck or the low back is considered one of the most frequent painful syndromes, a majority of which are self-limited. Various risk factors have been identified regarding these conditions such as age, obesity, inactivity (or in contrast, hard physical work) as well as smoking [7]. Our basic assumption is that, since the COVID-19 pandemic did not cause an essential change in most of the above factors, a significant difference in the rate of visits to the ED related these syndromes will not be observed. 
A marked reduction in ED visits during the lockdowns might suggest a possible abuse of the facility prior to the pandemic. A severe drop in the frequency of cardiologic and neurologic visits to the ED is especially vexing [8]. A report from Finland supports a marked decrease in ED consults regarding back and limb pain, but not concerning acute myocardial infarction during the early confinements of the COVID-19 pandemic [9]. A significant limitation in the number of presentations during lockdowns in the ED was interpreted as a lack of willingness to invest the necessary effort to acquire a medical facility if, and when required [10]. An evaluation of the number and rate of ED visits before the start of the COVID-19 pandemic was rare. One study, however [11], did rise to the challenge. Here, the number of ED visits after the start of the confinement displayed a marked reduction. In the present study, the majority of visits to the ED during the pandemic were due to LBP, mostly in patients older than 60 years of age. In contrast, most patients who visited ED at the beginning of the pandemic were between 40 to 60 years of age. From gender perspective: most women who visited the ED had LBP.

Consideration given to the number of weekly visits, as they occur before the epidemic or during its course (Table 1.), one observes a variance between the two eras, and that, to a significant degree! During COVID19 , patients suffering from back- or neck pain visited the ED in reduced numbers. It is not excluded that the above drop in presentation of the ED may represent a reduced morbidity from these disorders. But, another option is that, due to the lockdowns, individuals did indulge to a lesser degree in all forms of physical activity, including that practiced in closed spaces. Therefore, limited episodes of low-back- and neck-pain are expected, which request a less frequent rate of ED attendance. An alternative explanation may be, that, in a normalized period, the emergency facilities are freely attainable, and it is possible that a proportion of the visitors of the ED, do not precisely need the exam or the treatment, and such abuse might be practiced at times. Support to this thesis is displayed in the high variance between the low mean number of visits in the first quartile of the pandemic, as compared with the near-normal span that follows. Unfortunately, the above thesis is not anchored onto hard data. One is therefore invited to evaluate the proposed ideas, and to suggest some of his own, since speculations are not excluded hereby.

In contrast with the LBP patients, those complaining of NP were fewer. While there was a contraction in the absolute number, they represented a higher rate of ED visits (Tables 3,4$)$. The role that genders might have played in this investigation is probably limited. Low back pain is connected mainly with the preconfinement days, with age groups 40 to 60 . The time spent in ED by these patients is usually more than two hours (Table 4).

Figure 3 underlines the breakdown occurring during the transition from pre-lockdown into the first confinement. A marked drop in the number of visits to the ED may suggest that the effect described in relation with Table 1 might not be an artefact. During the first lockdown, many people had avoided hospitals, even when in serious need, out of fear of contamination. It is probable that during the later confinements the segregation between COVID-19 ED and general medical ED, was tighter. 
Two values in Table 2 are worth a discussion. One is the intercept, describing the rate of visits to the ED, one year before lockdown. The number of ED visits per week is high and the trend is positive. The second is the degree of change after the lockdown. The rate of visits per week is moderate, but the trend is negative. It appears that together with the musculoskeletal (MSK) symptoms, psychological and psychosocial complications become evident when the spine is predominantly affected. Thus, the inactivity and immobility brought about by a confinement may be at the origin of the symptoms. It has been stated previously that the predominant occurrence of LBP is overwhelming and is independent of whether a confinement is taking place. The lack of consequent genetic or environmental factors is suggested. The lockdown reflects immobility and a limitation regarding work activity $[13,14]$.

Overall, we confirm that more women than men visit the ED due to LBP. The causes have not been elucidated. One possible factor includes the hardship in sustaining quarantine, though it might be shared with lonely men. Visiting the ED offers the potential to socialize with other human beings. Hormonal factors should be taken in consideration, perhaps via the intermediary of calcium modulating hormones. Last, we will note that different emotional stressogenic causes may contribute to MSK pain of indefinite quality, which in some cases, are difficult to differentiate from a standard initiation of LBP. One should look at the COVID-19 pandemic's lockdowns, as a potential stimulators to such episodes.

\section{Limitations:}

The duration of 30 weeks suggested for the contrasted period associated with the compiled lockdown intervals, is not precisely estimated. The reason of this miscalculation originates both in a variable number of confinements, as well as in the changing nature of the lockdowns. While the first two sequences were strictly observed, this was not the case for the last two confinements.

\section{Conclusions}

Evidence of a higher rate of LBP, as well as of NP, to a lesser level, which may be associated indirectly with the COVID-19 pandemic is demonstrated. The association is generated by SARS-CoV-2, but rather as a result of the use of lockdowns as tools in the pandemic management. The confinements induced some degree of immobility and perhaps, stress, which contribute to MSK changes, essentially LBP. When the spine is involved, a further component prevails in the form of psychological/psychosocial deficits [12, 13]. Isolation, as a possible complication, might affect individuals and/or even core families, establishing a compound MSK-psychosocial clinical syndrome [14].

\section{Declarations}

The authors declare 'No conflict of interests exists'.

ACKNOWLEDGEMENTS: We thank Ari M.D. Klickstein, B.Sc., for his review of the manuscript.

\section{References}


1. Park KS (2020) Factors affecting awareness of myocardial infarction symptoms among the general public in Korea. Epidemiol Health 42:e2020032

2. Sederholm Lawesson S, Isaakson R-M, Thylen I et al (2018) Gender differences in symptom presentation of ST-elevation myocardial infarction - An observational multicenter survey study. Int J Cardiol 264:7-11

3. Hurwitz EL, Randhawa K, Yu H et al (2018) The Global Spine Care Initiative: a summary of thr global burden of low back pain and neck pain studies. Eur Spine J 27(Suppl 6):796-801

4. Caraceni A, Mendoza TR, Mencaglia E et al (1996) A validation study of an Italian version of the Brief Pain Inventory. Pain 65:87-92

5. Moretti A, Menna F, Aulicino M et al (2020) Characterization of Home Working Population during COVID-19 Emergency: A cross sectional analysis. Int J Environmental Res Public Health 17:6284

6. Lippi G, Henry BM, Bovo C et al (2020) Health risks and potential remedies during prolonged lockdowns for coronavirus disease 2019 (COVID-19). Diagnosis 7:85-90

7. Mulholland RH, Wood R, Stagg HR et al (2020) Impact of COVID-19 on accident and emergency attendances and emergency and planned hospital admissions in Scotlandd: an interrupted timeseries analysis. J Royal Soc Med 113:444-453

8. Fallon N, Brown C, Twiddy $\mathrm{H}$ et al (2021) Adverse effects of COVID-19-related lockdown on pain, physical activity, and psychological well-being in people with chronic pain. $\mathrm{Br} J$ Pain 15:357-368

9. Ojetti V, Covino M, Brigida M et al (2020) Non-COVID diseases during the pandemic: Where have all other emergencies gone? Medicina (Kaunas) 56:512

10. Kuitunnen I, Ponkilainen VT, Launonen AP et al (2020) The effect of national lockdown due to COVID19 on emergency department visits. Scand J Trauma Resusc Emergency Med 28:114

11. Tuominen J, Hallberg V, Oksala N et al (2020) NYU-EDA in modelling the effect of COVID-19 on patient volumes in a Finnish emergency department. BMC Emerg Med 20:97

12. Rennert-May E, Leal J, Thanh N-X et al (2021) The Impact of COVID-19 on hospital admissions and emergency department visits: a population-based study. PLoS ONE 16:e0252441

13. Chou R (2021) Low back pain. Ann Intern Med 174:ITC113-ITC128

14. Naliboff BD, Cohen MJ, Swanson GA et al (1985) Comprehensive association of chronic low back pain patients and controls: physical abilities, level of activity, psychological adjustment and pain perception. Pain 23:121-134

\section{Figures}




\section{Diagnosis in Emergency Department}

ICD Code

Back pain (BP)

$\begin{array}{ll}\text { SACROILIITIS, NOT ELSEWHERE CLASSIFIED } & 7202\end{array}$

$\begin{array}{ll}\text { LUMBAGO } & 7242\end{array}$

$\begin{array}{ll}\text { SCIATICA } & 7243\end{array}$

OTHER AND UNSPECIFIED DISORDERS OF BACK 124

$\begin{array}{ll}\text { LOW BACK PAIN } & 7242\end{array}$

DEGENERATION OF LUMBAR OR LUMBOSACRAL INTERVERTEBRAL DISC 72252

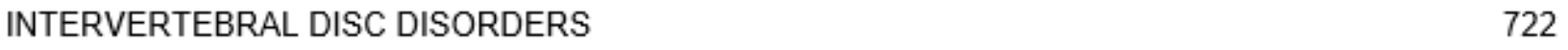

$\begin{array}{ll}\text { PAIN IN THORACIC SPINE } & 7241\end{array}$

$\begin{array}{ll}\text { DISC PROTRUSION } & 7220\end{array}$

HERNIATED INTERVERTEBRAL DISC LUMBAR SACRAL $\quad 7221$

DEGENERATION OF INTERVERTEBRAL DISC $\quad 7226$

Neck pain (NP)

$\begin{array}{ll}\text { TORTICOLLIS, UNSPECIFIED } & 7235\end{array}$

$\begin{array}{ll}\text { CERVICALGIA / NECK PAIN } & 7231\end{array}$

$\begin{array}{ll}\text { CERVICALGIA } & 7231\end{array}$

$\begin{array}{ll}\text { NECK PAIN } & 7231\end{array}$

$\begin{array}{ll}\text { OTHER DISORDERS OF CERVICAL REGION } & 723\end{array}$

$\begin{array}{ll}\text { CERVICAL PAIN } & 7231\end{array}$

$\begin{array}{ll}\text { CERVICAL SPONDYLOSIS WITH MYELOPATHY } & 7211\end{array}$

SPINAL STENOSIS IN CERVICAL REGION

Figure 1

A list of the diagnoses that were used to identified patients in the study group 


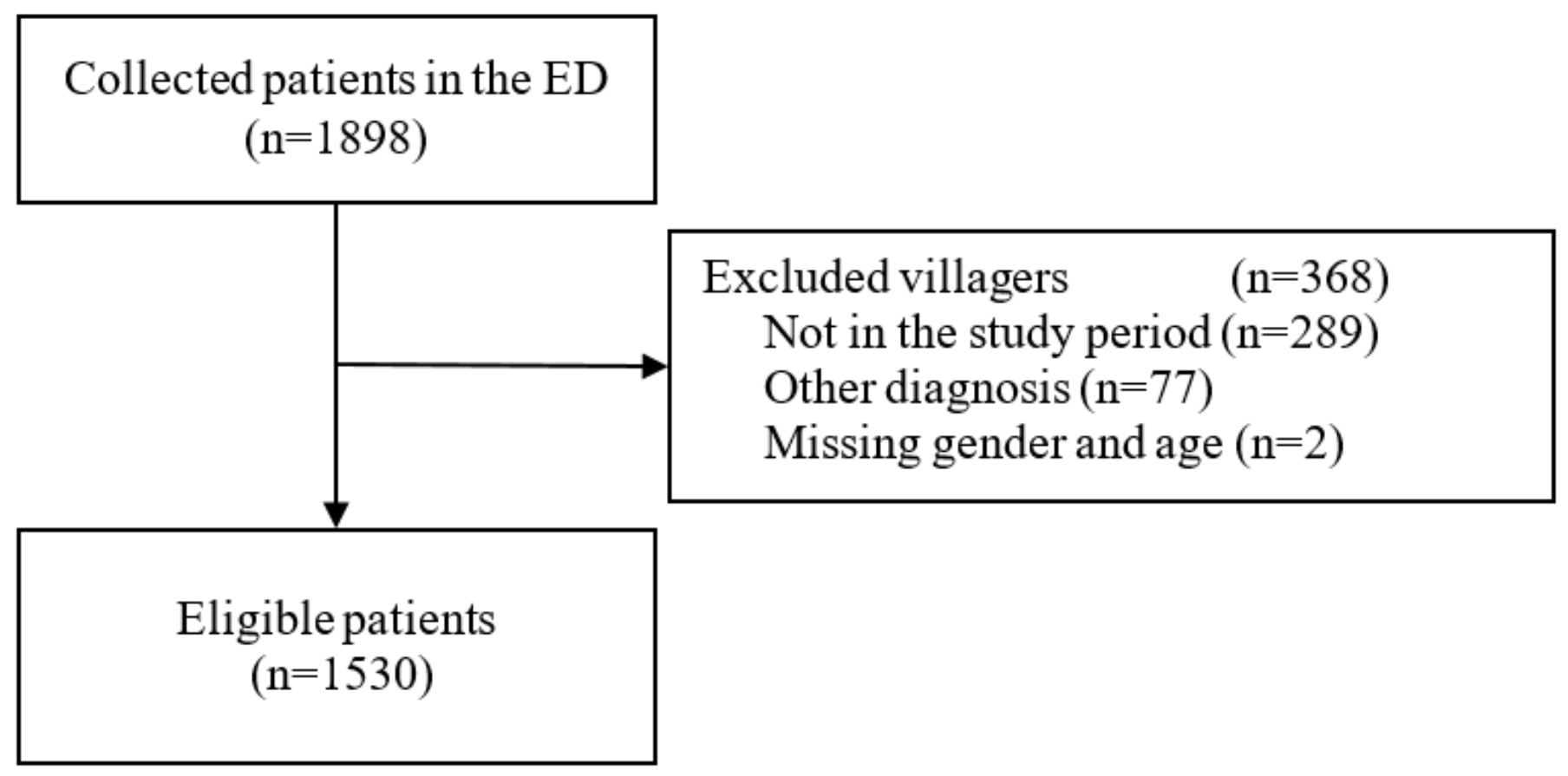

Figure 2

Flow diagram of eligible patients

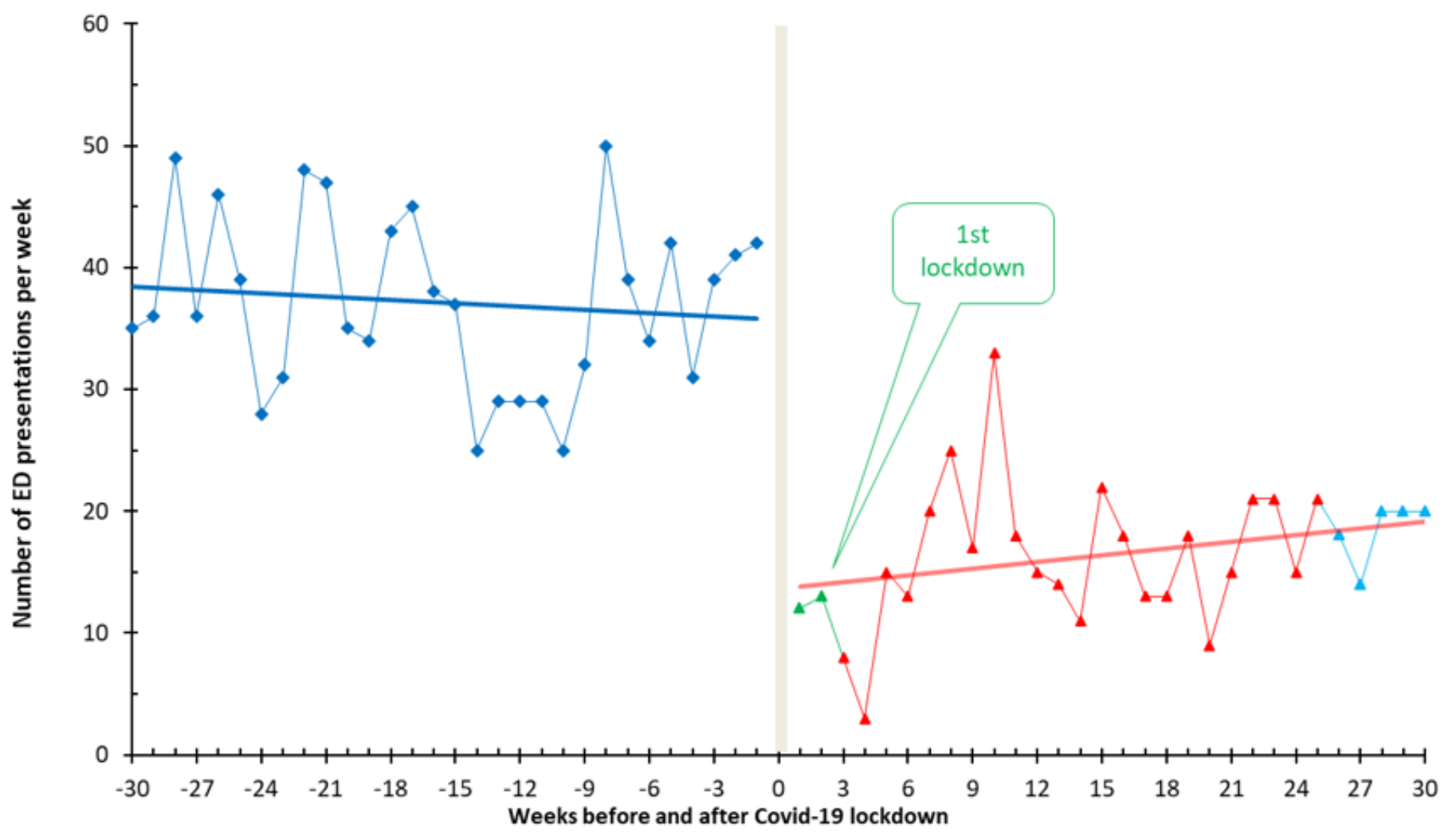

Figure 3 
Weekly number of emergency department presentations of eligible residents before and during lockdowns 